\title{
Cardiovascular surgery \\ GW23-e1573 SERUM LEVELS OF VON WILLEBRAND FACTOR ARE ASSOCIATED WITH ANGIOGRAPHIC ANGIOGRAPHIC NO-REFLOW AFTER PRIMARY PERCUTANEOUS CORONARY INTERVENTION FOR ST SEGMENT ELEVATION MYOCARDIAL INFARCTION
}

doi:10.1136/heartjnl-2012-302920u.1

${ }^{1} J$ un Yang, 'Lin Zhong, ${ }^{1}$ Xiang-ping Liu, ${ }^{1}$ Yi-min Fang, ${ }^{1}$ Fa-xin Ren, ${ }^{1}$ Chuanhuan Zhang, ${ }^{2}$ Jun Yang. 'Yantai Yuhuangding Hospital; ${ }^{2}$ Yantai Yuhuangding Hospital

Objectives Primary percutaneous coronary intervention (PPCI) is an important therapy for patients with acute ST-segment elevation myocardial infarction (STEMI). However, the restoration of epicardial coronary patency after coronary occlusion does not always guarantee adequate perfusion of myocardium at risk of ischaemia. This phenomenon was called no-reflow. It may inhibit the expected benefit from revascularisation of infarction related artery (IRA) and lead to poor functional and prognostic outcomes. The pathogenesis of no-reflow after PPCI is multifactorial, including distal embolisation from ruptured plaque or thrombus fragmentation, microvascular vasoconstriction, and, more importantly, platelet aggregation seems play a key role in the pathogenetic procedure. Therefore, mediators affecting platelet activation, such as von willebrand factor (vWF), might be involved in mechanism of noreflow. The aim of this study was to investigate whether serum level of vWF is associated with angiographic no-reflow and whether it could be one of the markers to predict angiographic noreflow after PPCI in patient with STEMI.

Methods 111 consecutive STEMI patients undergoing successful PPCI were studied. On the basis of post-stent TIMI flow and 
corrected TIMI frame count (CTFC) of target vessels, the patients were divided into two groups, namely no-reflow group $(n=25)$ and reflow group $(\mathrm{n}=86)$. The patients' venous blood sample was taken before and after PPCI (within $24 \mathrm{~h}$ ), and the serum levels of vWF, TnI, CK-MB, and hs-CRP were measured by enzyme linked immunosorbent assay (ELISA) and other methods. The differences between two groups were compared in basic clinical data, coronary angiography data, serum levels of vWF and other biomarkers. The association of vWF and other biomarkers with CTFC of target arteries were analysed. Multivariable logistic regression analysis was applied to identify independent clinical predictors for noreflow among the variables showing a significant association with no-reflow at univariate analysis.

Results In the comparison of basic clinical data, the two groups were similar with respect to sex, current smoking, hypertension, hyperlipidaemia, time from onset to taking blood, time of taking blood after PPCI, and asprin usage before admission $(p>0.05)$. While in the patients with angiographic no-reflow, the age was older, the incidence of diabetes mellitus was higher $(\mathrm{p}<0.05)$, LVEF on admission was lower $(54.8 \pm 9.1 \%$ vs $60.2 \pm 9.3 \%, p<0.05)$, and less statins taken $(31.7 \%$ vs $72.5 \%, p<0.01)$ when compared with reflow patients. According to coronary angiography data, there was significantly difference between the length of target lesion $(25.2 \pm 3.9 \mathrm{~mm}$ in no-reflow group vs $17.7 \pm 4.3 \mathrm{~mm}$ in reflow group, $\mathrm{p}<0.05)$, and the length of implanted stent $(30.8 \pm 3.7 \mathrm{~mm}$ in no-reflow group vs $23.4 \pm 4.1 \mathrm{~mm}$ in reflow group, $\mathrm{p}<0.05)$. There were no significantly difference in target vessel (LAD, LCX, RCA), bifurcational or calcified lesion, pre-procedure reference vessel diameter, pre-procedure minimal luminal diameter (MLD), pre-procedure stenosis, number of stent, time from onset to revascularisation. Before PPCI, the levels of serum vWF were significantly higher in no-reflow group than those in reflow group (153.7 $\pm 46.1 \mathrm{mU} / \mathrm{ml}$ vs $131.4 \pm 39.2 \mathrm{mU} / \mathrm{ml}, \mathrm{p}<0.05$ ). The serum levels of vWF after PPCI were higher than that before PPCI either in no-reflow group $(236.5 \pm 52.2 \mathrm{mU} / \mathrm{ml}$ vs $151.9 \pm 44.3 \mathrm{mU} / \mathrm{ml}, \mathrm{p}<0.01)$ or in reflow group $(181.3 \pm 41.7 \mathrm{mU} / \mathrm{ml}$ vs $129.9 \pm 38.6 \mathrm{mU} / \mathrm{ml}, \mathrm{p}<0.01)$, and the increasing percentage between post-and pre-PPCI was larger in no-reflow group than that in reflow group $(59.4 \pm 22.7 \%$ vs 40.1 $\pm 19.2 \%, p<0.01)$. vWF serum levels pre-PPCI and post-PPCI were both positively correlated with CTFC of target vessels $(r=0.545$, $\mathrm{p}<0.01$ and $\mathrm{r}=0.658, \mathrm{p}<0.01$; respectively). In multivariate logistic regression analysis, the variables such as age, diabetes history, length of target lesion, length of stent, basic statin usage, LVEF on admission, vWF serum level and TnI serum level, were included in the model, and the result revealed that vWF serum level before PPCI, basic usage of statins, the length of target lesion, LVEF on admission were independent risk factors for angiographic no-reflow after PPCI.

Conclusions The serum levels of vWF, both pre- PPCI and post-PPCI, were significantly associated with angiographic no-reflow. The vWF serum level on admission is an independent risk factors for angiographic no-reflow in the patients with acute STEMI. vWF may play an important role in the pathogenesis of no-reflow after PCI by mediating platelet adhesion and aggregation, and promoting acute thrombus formation. This research also suggests the importance of inhibiting platelets function in patients with acute STEMI for prevention and treatment of angiographic no-reflow after revascularisation. 